\title{
Análise biométrica de ganhos por seleção em população de milho pipoca de quinto ciclo de seleção recorrente ${ }^{1}$
}

\author{
Biometric analysis of selection gains in popcorn population of the fifth cycle of \\ recurrent selection
}

\author{
Ramon Macedo Rangel ${ }^{2}$, Antonio Teixeira do Amaral Júnior ${ }^{3 *}$, Leandro Simões Azeredo Gonçalves ${ }^{3}$, Silvério de \\ Paiva Freitas Júnior ${ }^{4}$ e Liliam Silvia Candido ${ }^{3}$
}

\begin{abstract}
Resumo - Com o intento de selecionar progênies superiores para a efetiva concentração de alelos favoráveis em uma população de quinto ciclo de seleção recorrente intrapopulacional em famílias de irmãos-completos de milho pipoca, avaliaram-se 200 famílias quanto a seis características agronômicas, incluindo rendimento de grãos e capacidade de expansão, em delineamento aleatorizado em blocos com duas repetições dentro de 'sets' em dois ambientes distintos: Colégio Estadual Agrícola Antônio Sarlo, em Campos dos Goytacazes, e Estação Experimental da PESAGRO-RIO, em Itaocara, Rio de Janeiro, em 2007/2008. Houve diferenças significativas para famílias/“sets" para todas as características, indicando a presença de variabilidade genética a ser explorada nos futuros ciclos. Na seleção das progênies superiores o índice de Mulamba e Mock resultou em ganhos proporcionais mais adequados para capacidade de expansão e rendimento de grãos, com valores respectivos de 6,01 e $8,53 \%$, na utilização de pesos econômicos arbitrários.
\end{abstract}

Palavras-chave - Milho pipoca. Seleção de plantas. Melhoramento genético.

\begin{abstract}
Aiming to select superior progenies for concentration of favorable alleles in a popcorn population on the fifth cycle of intrapopulation full-sib recurrent selection, two hundred progenies were evaluated for six agronomic traits, including grain yield and popping expansion, in randomized complete block design with two replications within sets, in two distinct environments: Colégio Estadual Agrícola Antônio Sarlo, in Campos dos Goytacazes, and Experimental Station of PESAGRORIO, located in Itaocara, Rio de Janeiro State, in 2007/2008. There were significant differences among families within sets in all traits, indicating the presence of genetic variability to be exploited in the future cycles. The utilized selection indexes were the Mulamba and Mock; and Smith and Hazel. The Mulamba and Mock index propitiated more elevated magnitudes of the predicted gains for most of the traits, including popping expansion and grain yield, with values of 6.01 and $8.53 \%$, respectively, utilizing arbitrary weight attributed by tentative.
\end{abstract}

Key words - Popcorn. Plant selection. Plant breeding.

\footnotetext{
*Autor para correspondência

${ }^{1}$ Recebido para publicação em 28/04/2010; aprovado em 15/03/2011

Parte da Tese de Doutorado do primeiro autor apresentada ao programa de Pós-Graduação em Genética e Melhoramento de Plantas/UENF

${ }^{2}$ Sygenta Seeds Ltda. Rod. BR 452, km 142, Uberlandia-MG, Brasil, 38.400-974, ramonmr@uenf.br

${ }^{3}$ Universidade Estadual do Norte Fluminense Darcy Ribeiro, UENF, Av. Alberto Lamego 2000, Campos dos Goytacazes-RJ, Brasil, 28.013-602,

amaraljr@uenf.br, 1sagrural@yahoo.com.br, lcandido@uenf.br

${ }^{4}$ Universidade Federa do Ceará, Campus Carirí, Av. Tenente Raimundo Rocha, Juazeiro do Norte-CE, Brasil, 63.000-000, silveriojr@uenf.br
} 


\section{Introdução}

O cultivo de milho pipoca tem exercido influência positiva em setores da economia nacional (BRUGNERA et al., 2003; MENDES DE PAULA et al., 2010; RANGEL et al., 2008; SCAPIM et al., 2006; SILVA et al., 2010a; SIMON et al., 2004; VIEIRA et al., 2009). Considerada uma cultura de elevada rentabilidade, seu produto final possui grande aceitação popular e, em muito, movimenta a economia informal. É presença garantida nas gôndolas dos mais diversos estabelecimentos comerciais do ramo de alimentos, seja nas grandes ou nas pequenas cidades do país.

Entretanto, o reduzido número de cultivares de milho pipoca que reúnem características agronômicas favoráveis e com alto índice de capacidade de expansão é considerado um dos principais entraves para cultura no país (FREITAS JÚNIOR et al., 2009; MENDES DE PAULA et al., 2010; MIRANDA et al., 2003; PEREIRA; AMARAL JÚNIOR, 2001; SILVA et al., 2010b). Nesse sentido, o desenvolvimento de programas de melhoramento de milho pipoca, que visem o desenvolvimento de populações melhoradas e/ou híbridos adaptados as condições brasileiras, são imprescindíveis para diminuir a dependência da importação da pipoca (LEONELLO et al., 2009; VILARINHO et al., 2002).

Neste contexto, a seleção recorrente é considerada uma importante estratégia para a recomendação de variedades melhoradas, tendo como exemplo a variedade de milho pipoca BRS Angela, da EMBRAPA/CNPMS (PACHECO et al., 2000). O emprego de seleção recorrente visa à obtenção de variedade melhorada, com os consequentes aumentos das frequências de alelos favoráveis, sobretudo para rendimento de grãos e capacidade de expansão, que são as características de maior importância comercial (AMARAL JÚNIOR et al., 2010; HALLAUER; MIRANDA FILHO, 1988; SCAPIM et al., 2002).

A Universidade Estadual do Norte Fluminense Darcy Ribeiro (UENF) vem desenvolvendo um programa de seleção recorrente com milho pipoca, visando o lançamento de variedades com características desejáveis e adaptadas às regiões Norte e Noroeste do Estado do Rio de Janeiro (FREITAS JÚNIOR et al., 2009; PEREIRA; AMARAL JÚNIOR, 2001). O programa iniciou-se com a avaliação do potencial da variedade de polinização aberta, UNB-2U (PEREIRA; AMARAL JÚNIOR, 2001). A população UNB-2U originou-se de seleção em um 'Composto indígena', que a ESALQ/USP doou a UNB de Brasília. Nesse sentido, gerou-se a população UNB-1, que foi cruzada com a variedade de milho pipoca Americana, cujas progênies selecionadas foram cruzadas com uma variedade de grãos amarelos e resistentes a helmintosporiose. Após dois ciclos de seleção massal, foi obtida uma população formada por plantas resistentes e grãos amarelos. Tal população foi retrocruzada três vezes com a variedade Americana, originando a população UNB-2U.

Constatado o potencial de seleção do UNB-2U, quatro ciclos de seleção recorrente foram realizados, com a seleção de ganhos de produtividade e de capacidade de expansão de, respectivamente, $10,39 \%$ e $4,69 \%$ no primeiro ciclo (DAROS et al., 2004a), 26,95\% e 17,80\% no segundo ciclo (DAROS et al., 2004b), 10,00\% e 7,16\% no terceiro ciclo (SANTOS et al., 2007) e de 7,71\% e 10,58\% no quarto ciclo (AMARAL JÚNIOR et al., 2010; FREITAS JÚNIOR et al., 2009).

O presente trabalho teve por objetivo estimar os ganhos previstos para o quinto ciclo de seleção recorrente para características de interesse ao programa de melhoramento de milho-pipoca da UENF.

\section{Material e métodos}

Foi empregado o método de seleção recorrente pela obtenção de 200 famílias de irmãos completos. As progênies foram avaliadas em dois ambientes distanciados a $120 \mathrm{~km}$, no Estado do Rio de Janeiro: Campo Experimental da UENF, anexo ao Colégio Estadual Agrícola Antônio Sarlo, em Campos dos Goytacazes, com altitude de 10 m, e Campo Experimental da UENF em Itaocara - Ilha Barra do Pomba - com $90 \mathrm{~m}$ de altitude, respectivamente, Regiões Norte e Noroeste Fluminense. Em cada ensaio, foram utilizadas como testemunhas os híbridos IAC 112, IAC 125 e Zélia.

O delineamento utilizado foi blocos aleatorizados com duas repetições dentro de 'sets'. As 200 famílias e as testemunhas foram arranjadas em oito 'sets'. Assim, cada 'set' conteve 25 famílias e três testemunhas, totalizando 28 tratamentos. As parcelas experimentais foram constituídas por fileiras simples de $5,00 \mathrm{~m}$ de comprimento, com espaçamento de $0,90 \mathrm{~m}$ ente linhas e $0,20 \mathrm{~m}$ entre plantas, totalizando $55.555 \mathrm{pl} \mathrm{ha}^{-1}$. Foram semeadas três sementes por cova a uma profundidade de $0,05 \mathrm{~m}$ e, aos 21 dias após a emergência, realizados o desbaste, deixando-se uma planta por cova.

De acordo com a análise de solo, a adubação de semeadura foi realizada aplicando-se $800 \mathrm{~kg} \mathrm{ha}^{-1}$ de adubo N-P-K na formulação 04-14-08. A primeira adubação de cobertura foi realizada 30 dias após a semeadura, por ocasião da amontoa, aplicando-se 300 $\mathrm{kg} \mathrm{ha}^{-1}$ da formulação 20-00-20. A segunda adubação foi realizada aos 45 dias após a semeadura na dosagem de $260 \mathrm{~kg} \mathrm{ha}^{-1}$ de sulfato de amônio (SAWAZAKI, 2001). A colheita manual das espigas foi realizada no mês de março de 2008. 


\section{As características agronômicas avaliadas foram:}

- Altura de planta (AP) - expressa em cm, sendo a média da parcela considerado a distância do nível do solo à inserção da folha bandeira em seis plantas competitivas;

- Altura de inserção da primeira espiga (AE) - também expressa em cm, sendo a média da parcela, e mensurada nas mesmas plantas utilizadas para a característica anterior, compreendendo a distância entre o nível do solo e a base de inserção da espiga superior;

- Número de espigas doentes (ED) - obtido pela contagem de espigas doentes em cada parcela na ocasião da colheita;

- Número de espigas atacadas por pragas (EP) - expresso pela contagem de espigas atacadas por pragas em cada parcela na ocasião da colheita;

- Rendimento de grãos (RG) - obtido por pesagem dos grãos em cada parcela, após debulha, e expresso em $\mathrm{kg} \mathrm{ha}^{-1}$; e

- Capacidade de expansão (CE) - expressa pela relação $\mathrm{mL} \mathrm{g}^{-1}$, ou seja, volume de pipoca estourada em relação à massa de grãos submetida ao pipocamento. A massa de grãos submetida ao pipocamento compreendia 30 g e o volume resultante da expansão foi mensurado em proveta de $2.000 \mathrm{~mL}$. Para a quantificação da capacidade de expansão, foi utilizado um aparelho de microondas da marca Panasonic modelo NNS65B, sob potência máxima de 1.000 watts durante 3 minutos, com duas subamostragens em cada parcela. Os grãos foram colocados dentro de potes apropriados para estourar pipoca (Corn Popper, da Nordic Ware), sem óleo.

Para posterior recombinação das famílias selecionadas, uma quantidade suficiente de sementes remanescentes de todas as famílias de irmãos completos ficou armazenada.

Os dados das características foram utilizados para a realização da análise de variância de acordo com o modelo estatístico $\mathrm{Y}_{\mathrm{ijkl}}=\mu+\mathrm{A}_{\mathrm{i}}+\mathrm{S}_{\mathrm{j}}+\mathrm{AS}_{\mathrm{ij}}+\mathrm{R} / \mathrm{AS}_{\mathrm{ijk}}+\mathrm{F} / \mathrm{S}_{\mathrm{jl}}+$ $\mathrm{AF} / \mathrm{S}_{\mathrm{ijl}}+\xi_{\mathrm{ijkl},}$, em que: $\mathrm{Y}_{\mathrm{ijkl}}$ é o valor fenotípico médio da parcela; $\mu$ é a média; A é o efeito fixo do i-ésimo ambiente; $\mathrm{S}_{\mathrm{j}}$ é o efeito do j-ésimo "set"; $\mathrm{AS}_{\mathrm{ij}}$ é o efeito da interação entre ambientes e "sets"; $\mathrm{R} / \mathrm{AS}_{\mathrm{ijk}}$ é o efeito da k-ésima repetição dentro da interação entre o i-ésimo ambiente e o j-ésimo "set"; $F / S_{j 1}$ é o efeito aleatório do i-ésimo genótipo dentro do j-ésimo "set"; $A F / S_{i j 1}$ é o efeito da interação de ambientes e genótipos dentro do j-ésimo "set" e $\xi_{\mathrm{ijkl}}$ é o erro experimental. Foram obtidas as estimativas dos componentes de variância, sendo o estimador da variância genotípica entre famílias expresso por:

$$
\hat{\sigma}_{G}^{2}=\frac{Q M S / S-Q M R}{a r}
$$

em que: $Q M S / S$ : quadrado médio de famílias dentro de "sets"; $Q M R$ : quadrado médio do resíduo; $r$ : repetição e a: ambiente. A variância fenotípica e $a$ variância residual foram expressas, respectivamente, pelas seguintes equações:

$\hat{\sigma}_{F}^{2}=\frac{Q M F / S}{a r} \quad$ e $\quad \hat{\sigma}_{r}^{2}=\frac{Q M R}{a r}$

A variância da interação genótipo "versus" ambiente foi determinado pela equação:

$\hat{\sigma}_{G A}^{2}=\frac{Q M\left(A^{*} F\right) / S-Q M R}{r} \cdot \frac{a-1}{a}$

sendo $Q M\left(A^{*} F\right) / S$ : quadrado médio da interação Famílias "versus" Ambiente dentro de 'Sets'. A herdabilidade com base na média de famílias foi estimada pela expressão:

$\hat{h}_{\bar{x}}^{2}=\frac{\hat{\sigma}_{G}^{2}}{\hat{\sigma}_{F}^{2}}=\frac{Q M F / S-Q M R}{Q M F / S}$

O coeficiente de variação genético percentual e o índice de variação foram obtidos segundo Cruz e Carneiro (2003), empregando-se, respectivamente as seguintes equações:

$C v_{g}=100 \sqrt{\frac{\sigma_{g}^{2}}{\bar{X}}}$ e $\quad \hat{I}_{v}=\frac{C V g}{C V e}$

As predições dos ganhos por seleção foram obtidas pelos índices de Mulamba e Mock (1978) e de Smith (1936) e Hazel (1943).

O índice de Mulamba e Mock (1978) hierarquiza os genótipos, inicialmente, para cada característica, por meio da atribuição de valores absolutos mais elevados àqueles de melhor desempenho. Por fim, os valores atribuídos a cada característica são somados, obtendo-se a soma dos "ranks", que assinala a classificação dos genótipos. O índice de Smith (1936) e Hazel (1943) fundamenta-se na solução do sistema matricial: $\mathrm{b}=P^{-1} G a$, em que b é o vetor de dimensão $6 \times 1$ dos coeficientes de ponderação do índice a serem estimados; $P^{-1}$ é a inversa da matriz de dimensão 6 x 6 de variância e covariância fenotípicas entre as características; $G$ é a matriz de dimensão 6 x 6 de 
variâncias e covariâncias genéticas entre as características e $a$ é um vetor $6 \times 1$ de pesos econômicos.

\section{Resultados e discussão}

No que se refere ao efeito de ambientes, somente não houve diferença significativa $(\mathrm{P}>0,05) \mathrm{em}$ relação à capacidade de expansão (CE). Para as demais características, a significância demonstra que os ambientes foram suficientemente distintos para propiciar a verificação de diferenças entre as características (TAB. 1).

Para a fonte de variação Famílias dentro de 'Set' (F/S), com exceção de EP, que expressou significância em nível de 0,05 , as demais características revelaram significância $(\mathrm{P} \leq 0,01)$, denotando haver suficiente variabilidade genética a ser explorada em ciclos futuros de seleção.

Segundo Paterniani (1980), a variabilidade genética, o método de seleção empregado, o tamanho da população e a influência do ambiente são fatores que interferem na taxa de elevação das frequências gênicas favoráveis como efeito da seleção. $\mathrm{O}$ aumento nas frequências de alelos favoráveis na população melhorada a cada ciclo de seleção recorrente, traz como consequência o incremento da média populacional para diversas características (HALLAUER; MIRANDA FILHO, 1988). Neste contexto, a implementação do quinto ciclo de seleção recorrente entre famílias de irmãos completos resultou em êxito ao manter a variabilidade genética e incrementar de forma favorável as médias das características (AMARAL JÚNIOR et al., 2010; DAROS et al., 2004a; DAROS et al., 2004b; FREITAS JÚNIOR et al., 2009; SANTOS et al., 2007).

A significância de CE para a interação Ambiente "versus" Famílias dentro de 'Set' revela que as famílias comportaram-se de forma dissimilar em relação às mudanças das condições edafoclimáticas, estando em consonância com o preconizado por Brunson (1937) de que a CE é influenciada pelo ambiente. Conforme Robbins e Ashman (1984), a influência do ambiente na capacidade de expansão do milho pipoca é justificada pelo fato de que nem todos os genes que contribuem para dureza do endosperma contribuem para a capacidade de expansão dos grãos.

Os maiores valores de coeficiente de variação foram expressos para as características EP e ED, respectivamente, $60,79 \%$ e $60,73 \%$, sendo classificados como muito altos (GOMES, 2000). Freitas Júnior et al. (2009), na condução do quarto ciclo de seleção recorrente com esta população, também verificaram que os maiores valores de CVe estavam associados a estas mesmas características e concluiu sê-las de maior sensibilidade em relação à influência ambiental. Cargnelutti Filho e Storck (2007) relatam que é comum o descarte de características com

Tabela 1 - Estimativas dos quadrados médios, das médias e dos coeficientes de variação experimental de seis características avaliadas em 200 famílias de irmãos completos de milho pipoca de quinto ciclo de seleção recorrente. Campos dos Goytacazes e Itaocara-RJ, 2008

\begin{tabular}{|c|c|c|c|c|c|c|c|}
\hline \multirow{2}{*}{ FV } & \multirow{2}{*}{ GL } & \multicolumn{6}{|c|}{ Quadrados Médios $^{1 /}$} \\
\hline & & AP & $\mathrm{AE}$ & ED & EP & RG & $\mathrm{CE}$ \\
\hline Ambiente (A) & 1 & $242.733,76^{* *}$ & $42.945,01^{* *}$ & $2.099,52^{* *}$ & $2.106,00^{* *}$ & $121.193 .385,30^{* *}$ & $12,44^{\text {ns }}$ \\
\hline Set $(S)$ & 7 & $804,84^{\mathrm{ns}}$ & $187,23^{\mathrm{ns}}$ & $11,80^{\mathrm{ns}}$ & $28,05^{\mathrm{ns}}$ & $1.959 .249,90^{* *}$ & $53,80^{\text {ns }}$ \\
\hline $\mathrm{A} \times \mathrm{S}$ & 7 & $886,33^{\text {ns }}$ & $334,09^{\text {ns }}$ & $7,10^{\mathrm{ns}}$ & $24,87^{\mathrm{ns}}$ & $955.050,00^{\text {ns }}$ & $18,94^{\mathrm{ns}}$ \\
\hline $\operatorname{Rep}(\mathrm{R}) / \mathrm{A} \times \mathrm{S}$ & 16 & $1541,92^{* *}$ & $669,94^{* *}$ & $20,27^{* *}$ & $13,05^{\mathrm{ns}}$ & $824.373,60^{* *}$ & $7,95^{\mathrm{ns}}$ \\
\hline Famílias (F)/ S & 192 & $668,46^{* *}$ & $432,80^{* *}$ & $12,64^{* *}$ & $18,40^{*}$ & $687.512,10^{* *}$ & $51,76^{* *}$ \\
\hline$(\mathrm{A} \times \mathrm{F}) / \mathrm{S}$ & 192 & $158,36^{* *}$ & $93,11^{* *}$ & $10,42^{* *}$ & $13,42^{\mathrm{ns}}$ & $292.208,90^{\text {ns }}$ & $6,36^{*}$ \\
\hline Resíduo & 384 & 115,87 & 66,83 & 7,13 & 14,74 & $257.973,20$ & 4,82 \\
\hline Média & & 194,56 & 120,75 & 4,40 & 6,32 & $2.569,70$ & 26,27 \\
\hline $\mathrm{CVe}(\%)$ & & 5,53 & 6,77 & 60,73 & 60,79 & 19,76 & 8,33 \\
\hline
\end{tabular}

AP: altura de planta em cm; AE: altura de inserção da primeira espiga em cm; ED: número de espigas doentes; EP: número de espigas atacadas por pragas; RG: rendimento de grãos em $\mathrm{kg} \mathrm{ha}^{-1}$; e CE: capacidade de expansão em $\mathrm{mL} \mathrm{g}^{-1}$; $^{* *}$ : Significativo $(\mathrm{P} \leq 0,01)$ pelo teste F; ${ }^{*}$ : Significativo $(\mathrm{P} \leq 0,05)$ pelo teste $\mathrm{F} ; \mathrm{e}^{\mathrm{ns}}$ : Não Significativo 
baixa precisão em experimentos, e que o problema em se decidir pelo descarte ou não está nos casos em que a precisão é muito baixa (CVe muito alto) e a característica é de efeito não significativo para tratamento, o que neste trabalho não se aplica, em virtude da significância para EP e ED quanto a Famílias dentro de 'Set'.

Em programas de seleção recorrente é importante avaliar não apenas as estimativas das médias da população original, da população selecionada e das testemunhas, mas também os limites inferiores e superiores das médias das famílias avaliadas, sobretudo para as características de maior importância econômica, como CE e RG.

Santos et al. (2007), na condução da população de terceiro ciclo de seleção recorrente, identificaram para CE magnitudes de $10,33 \mathrm{~mL} \mathrm{~g}^{-1}$ e $39,67 \mathrm{~mL} \mathrm{~g}^{-1}$, respectivamente, para limite inferior e superior. Freitas Júnior et al. (2009), com base na avaliação do quarto ciclo, constataram magnitudes de $11,20 \mathrm{~mL} \mathrm{~g}^{-1}$, para o limite inferior, e $35,90 \mathrm{~mL} \mathrm{~g}^{-1}$, para o limite superior. No presente trabalho, tais valores foram de $15,17 \mathrm{~mL} \mathrm{~g}^{-1} \mathrm{e}$ $34,34 \mathrm{~mL} \mathrm{~g}^{-1}$, respectivamente. Esta redução na amplitude da variação não configura perda de variabilidade ao longo dos ciclos, já que houve diferença para Famílias dentro de 'Sets' quanto à característica CE (TAB. 1), de forma semelhante ao ocorrido no terceiro e quarto ciclos de seleção desta população (FREITAS JÚNIOR et al., 2009; SANTOS et al., 2007).

Não se deve desconsiderar que a predominância de efeitos genéticos aditivos para CE (RANGEL et al., 2008; SIMON et al., 2004) e elevadas magnitudes de herdabilidade (PEREIRA; AMARAL JÚNIOR, 2001), associadas ao avanço das gerações de seleção e recombinação, podem estar contribuindo para a redução da amplitude de variação quanto à capacidade de expansão e concorrendo para o estabelecimento de um nível de pipocamento adequado à população de quinto ciclo em virtude do acúmulo de alelos aditivos favoráveis.

A averiguação dos limites inferiores e superiores também tem sido realizada em outras populações sob seleção, como ocorreu com CMS-42 e CMS-43, no segundo ciclo de seleção recorrente, em que Pacheco et al. (1998) se fundamentaram nestes limites para predizerem a perspectiva de obtenção de segregantes superiores para CE e RG.

Ao se comparar as médias de RG das famílias avaliadas neste estudo, em relação aos últimos três ciclos, verifica-se que estas foram, respectivamente, $1.135,58 \mathrm{~kg} \mathrm{ha}^{-1}$ (SANTOS et al., 2007), 2.393,98 kg ha-1 (FREITAS JÚNIOR et al., 2009) e 2.569,70 kg ha-1 (TAB. 2), o que está em consonância com o propósito da seleção recorrente de aumento na frequência de alelos favoráveis e, consequentemente, incremento nas médias preditas após cada ciclo de seleção. O mesmo ocorreu com a CE, com magnitudes de 23,30 $\mathrm{mL} \mathrm{g}^{-1}$ (SANTOS et al., 2007), 25,06 $\mathrm{mL} \mathrm{g}^{-1}$ (FREITAS JÚNIOR et al., 2009) e 26,27 $\mathrm{mL} \mathrm{g}^{-1}$ (TAB. 2).

As estimativas das médias das testemunhas foram inferiores a das famílias avaliadas para a maioria das características, excetuando-se AP, AE, EP e CE. Para as três primeiras características, estes resultados revelam uma situação favorável ao melhoramento da população de milho pipoca avaliada neste estudo, indicando que as famílias apresentaram menor quebramento de plantas e melhor estande, empalhamento, sanidade e rendimento de grãos. Para CE, os valores das testemunhas, de $27,54 \mathrm{~mL} \mathrm{~g}^{-1}$, não

Tabela 2 - Estimativas das médias da população original $\left(\bar{X}_{o}\right)$, da população selecionada ${ }^{1 /}\left(\bar{X}_{s}\right)$ e das testemunhas $\left(\bar{X}_{T}\right)$; bem como os limites inferiores (LI) e superiores (LS) das médias das famílias de irmãos completos avaliados quanto a seis características ${ }^{2 /}$ no quinto ciclo de seleção recorrente da população UENF de milho pipoca. Campos dos Goytacazes e Itaocara-RJ, 2008

\begin{tabular}{|c|c|c|c|c|c|}
\hline Características & $\bar{X}_{o}$ & $\bar{X}_{s}$ & $\bar{X}_{T}$ & $\mathrm{LI}$ & $\mathrm{LS}$ \\
\hline AP & $194,55( \pm 9,73)$ & $192,77( \pm 6,63)$ & $193,96( \pm 4,33)$ & 164,80 & 273,07 \\
\hline $\mathrm{AE}$ & $120,74( \pm 8,43)$ & $120,58( \pm 5,14)$ & $116,17( \pm 3,12)$ & 89,80 & 147,90 \\
\hline ED & $4,39( \pm 0,35)$ & $4,02( \pm 0,11)$ & $6,86( \pm 0,23)$ & 1,00 & 10,25 \\
\hline EP & $6,31( \pm 0,53)$ & $6,22( \pm 0,33)$ & $6,06( \pm 0,19)$ & 1,75 & 13,25 \\
\hline $\mathrm{RG}$ & $2.569,70( \pm 326,67)$ & $2.920,93( \pm 198,89)$ & $1.969,23( \pm 142,27)$ & $1.466,66$ & $4.038,89$ \\
\hline $\mathrm{CE}$ & $26,27( \pm 3,29)$ & $29,30( \pm 1,96)$ & $27,54( \pm 1,14)$ & 15,17 & 34,34 \\
\hline
\end{tabular}


foram sobremaneira elevados ao das famílias avaliadas, com magnitude de 26,27 $\mathrm{mL} \mathrm{g}^{-1}$ (TAB. 2).

As estimativas da variância fenotípica $\left(\hat{\sigma}_{F}^{2}\right)$, genotípica $\left(\hat{\sigma}_{G}^{2}\right)$ e residual $\left(\hat{\sigma}_{r}^{2}\right)$, bem como da herdabilidade com base na média de famílias $\left(\hat{h}_{\hat{\imath}}^{2}\right)$, do coeficiente de variação genético ( $\hat{C V} g)$ e do índice de variação $\left(\hat{I}_{v}\right)$, nas características avaliadas, revelaram elevadas estimativas das variâncias genotípicas para as características AP, AE, RG e CE, acompanhadas de valores de herdabilidade com base na média de famílias, acima de $60 \%$, e índice de variação com magnitudes superiores a 0,60 (TAB. 3). Assim, há possibilidade de identificação de famílias ou genótipos superiores para as características mencionadas, sobretudo quanto a rendimento de grãos e capacidade de expansão.

As maiores magnitudes para as estimativas da herdabilidade e do índice de variação foram expressas pela CE, com valores respectivos de $90,68 \%$ e $1,56 \%$ (TAB. 3 ). Estes resultados corroboram os obtidos por Pereira e Amaral Júnior (2001), empregando o Delineamento I (COMSTOCK; ROBINSON, 1948), quando dos estudos iniciais com a população-base, a priori da aplicação dos ciclos de seleção.

Daros et al. (2002), na condução do primeiro ciclo de seleção recorrente com famílias de irmãos-completos, obtiveram estimativa de herdabilidade para $\mathrm{CE}$ igual a $77,75 \%$. Na condução do segundo ciclo, com famílias endogâmicas, Daros et al. (2004b) detectaram valor de $82,91 \%$. Santos et al. (2007), no terceiro ciclo, trabalhando com famílias de meios-irmãos, obtiveram estimativa de 68,41\%. Freitas Júnior et al. (2009) avaliaram famílias de irmãos-completos e obtiveram estimativa de 90,19\%. No presente ciclo, a estimativa de herdabilidade foi de $90,68 \%$. Com exceção do terceiro ciclo, no qual houve redução na estimativa da herdabilidade, provavelmente atribuída à influência ambiental (SANTOS et al., 2007), observa-se um aumento progressivo e significativo quanto à magnitude dos valores, ratificando a predominância de efeitos genéticos aditivos na expressão da característica (FREITAS JÚNIOR et al., 2009; SCAPIM et al,. 2002, SIMON et al., 2004).

Para rendimento de grãos, os valores de herdabilidade ao longo dos ciclos foram, respectivamente, 57,48\% (DAROS et al., 2004a), 84,15\% (DAROS et al., 2004b), $50,17 \%$ (SANTOS et al., 2007) e 56,88\% (FREITAS JÚNIOR et al., 2009). Estes percentuais foram superiores aos estimados para esta população por Pereira e Amaral Júnior (2001), com magnitude de 44,88\% para ganhos na população-base.

No presente ciclo, a estimativa de ganhos seletivos para $\mathrm{RG}$ foi de $62,47 \%$ (TAB. 3), revelando aumento na concentração de alelos favoráveis na população melhorada a cada ciclo de seleção recorrente (HALLAUER; MIRANDA FILHO, 1988). Embora RG tenha maior predominância de efeitos gênicos dominantes (SIMON et al., 2004), a manifestação da ação gênica aditiva tem sido observada para a característica (PEREIRA; AMARAL JÚNIOR 2001; SCAPIM et al., 2002).

As superioridades dos valores de herdabilidade para CE em relação a $\mathrm{RG}$ eram esperados ocorrerem na população avaliada, conforme preconizado por Pereira e Amaral Júnior (2001), por meio do Delineamento I, na populaçãobase. Pacheco et al. (1998), avaliando as populações CMS42 e CMS-43 de milho pipoca de segundo ciclo de seleção recorrente, obtiveram estimativas de herdabilidade para

Tabela 3 - Estimativas da variância fenotípica $\left(\hat{\sigma}_{F}^{2}\right)$, da variância genotípica $\left(\hat{\sigma}_{G}^{2}\right)$, da variância residual $\left(\hat{\sigma}_{r}^{2}\right)$, da variância da interação genótipo "versus" ambiente $\left(\hat{\sigma}_{G A}^{2}\right)$, da herdabilidade com base na média de famílias $\left(\hat{h}_{\bar{\gamma}}^{2}\right)$, do coeficiente de variação genético $(\hat{C V g})$ e do índice de variação $\left(\hat{I}_{v}\right)$, para seis características avaliadas em 200 famílias de irmãos completos no quinto ciclo de seleção recorrente na população UENF de milho pipoca. Campos dos Goytacazes e Itaocara-RJ, 2008

\begin{tabular}{crrrrrrr}
\hline Características $^{1 /}$ & \multicolumn{1}{c}{$\hat{\sigma}_{F}^{2}$} & \multicolumn{1}{c}{$\hat{\sigma}_{G}^{2}$} & \multicolumn{1}{c}{$\hat{\sigma}_{r}^{2}$} & \multicolumn{1}{c}{$\hat{\sigma}_{G A}^{2}$} & $\hat{h}_{\bar{x}}^{2}$ & \multicolumn{1}{c}{$\hat{C V g}$} & $\hat{I}_{v}$ \\
\hline $\mathrm{AP}$ & 167,11 & 138,14 & 28,96 & 10,62 & 82,66 & 6,04 & 1,09 \\
$\mathrm{AE}$ & 108,20 & 91,49 & 16,70 & 6,57 & 84,55 & 7,92 & 1,17 \\
$\mathrm{ED}$ & 3,16 & 1,37 & 1,78 & 0,82 & 43,60 & 26,69 & 0,43 \\
$\mathrm{EP}$ & 4,60 & 0,91 & 3,68 & 0,01 & 19,88 & 15,14 & 0,24 \\
$\mathrm{RG}$ & $171.878,02$ & $107.384,72$ & $64.493,30$ & $8.558,92$ & 62,47 & 12,75 & 0,64 \\
$\mathrm{CE}$ & 12,94 & 11,73 & 1,20 & 0,38 & 90,68 & 13,04 & 1,56 \\
\hline
\end{tabular}

${ }^{1 /}$ AP: altura de planta em cm; AE: altura de inserção da primeira espiga em cm; ED: número de espigas doentes; EP: número de espigas atacadas por pragas; RG: rendimento de grãos em $\mathrm{kg} \mathrm{ha}^{-1}$; e CE: capacidade de expansão em $\mathrm{mL} \mathrm{g}^{-1}$ 
capacidade de expansão com percentuais $27,40 \%$ e $42,90 \%$, e, para rendimento, de $57,60 \%$ e $60,10 \%$, respectivamente.

A comparação das estimativas dos parâmetros genéticos (TAB. 3) com os obtidos por Pacheco et al. (1998) na avaliação de CMS-42, que originou a variedade BRSAngela, ratificam a possibilidade de ganhos genéticos com a seleção para ambas as características, tendo em vista o intento de recomendação de uma nova cultivar para o Norte e Noroeste Fluminense. Quanto a ED e EP, em virtude dos valores de $\hat{I}_{v}$ inferiores a 0,50 e baixas magnitudes de herdabilidade, progressos genéticos através das gerações por meio de métodos simples de melhoramento não são muito promissores. Estas características expressaram estimativas de variâncias residuais superiores às genotípicas, indicando sê-las altamente influenciadas pelo ambiente. De modo especial para ED, a ocorrência da interação Ambiente "versus" Famílias dentro de 'Set' (TAB. 1) pode ser atribuída à forte influência do ambiente.

O índice de Mulamba and Mock (1978) revelou ganhos proporcionais mais adequados para o conjunto de características avaliadas empregando-se pesos econômicos atribuídos por tentativas (PT) (TAB. 4), corroborando resultados deste índice obtidos com ciclos anteriores desta população por Santos et al. (2007) e Freitas Júnior et al. (2009).

Os ganhos preditos mais expressivos para capacidade de expansão $(6,01 \%)$ e rendimento de grãos $(8,53 \%)$ foram obtidos empregando-se pesos econômicos atribuídos por tentativas (PT). O uso de pesos econômicos atribuídos por tentativas permitiu não apenas ganhos satisfatórios para RG e CE, mas também para as demais características avaliadas, quais sejam: (AP), com magnitude de $-0,76 \%$; altura de inserção da primeira espiga (AE), com -0,12\%; número de espigas doentes (ED), com $-3,68 \%$ e número de espigas atacadas por pragas (EP), com -0,29\% (TAB. 4). Os ganhos negativos para AP e $\mathrm{AE}$ foram satisfatórios devido à necessidade em reduzir o porte das plantas de milho frente aos fortes ventos que ocorrem nas regiões Norte e Noroeste Fluminense.

Arnhold e Viana (2007), trabalhando com a população de milho pipoca Beija Flor, visando à obtenção de linhagens, também encontraram maiores perspectivas

Tabela 4 - Estimativas de ganhos percentuais, por diferentes índices de seleção e pesos econômicos ${ }^{1 /}$, em seis características ${ }^{2 /}$ avaliadas em 200 famílias de irmãos completos, no quinto ciclo de seleção recorrente na população UENF de milho pipoca. Campos dos Goytacazes e Itaocara-RJ, 2008

\begin{tabular}{|c|c|c|c|c|c|}
\hline \multirow{2}{*}{ Características } & \multicolumn{5}{|c|}{ Mulamba e Mock } \\
\hline & DPg & $\mathrm{CVg}$ & Iv & $h^{2}$ & PT \\
\hline AP & 2,13 & $-1,00$ & $-5,06$ & $-4,27$ & $-0,76$ \\
\hline $\mathrm{AE}$ & 3,29 & $-0,21$ & $-5,77$ & $-4,38$ & $-0,12$ \\
\hline ED & $-2,61$ & $-8,05$ & $-2,03$ & $-1,79$ & $-3,68$ \\
\hline EP & 0,83 & $-0,42$ & $-0,21$ & $-0,55$ & $-0,29$ \\
\hline $\mathrm{RG}$ & 16,11 & 5,44 & 2,88 & 4,38 & 8,53 \\
\hline $\mathrm{CE}$ & 0,30 & 2,32 & 2,24 & 3,17 & 6,01 \\
\hline \multirow{2}{*}{ Características } & \multicolumn{5}{|c|}{ Smith e Hazel } \\
\hline & DPg & $\mathrm{CVg}$ & Iv & $h^{2}$ & PT \\
\hline AP & 2,65 & 2,83 & 3,45 & 3,25 & 2,65 \\
\hline $\mathrm{AE}$ & 3,72 & 4,08 & 4,81 & 4,24 & 4,48 \\
\hline ED & $-4,18$ & $-4,50$ & $-5,08$ & $-4,50$ & $-5,25$ \\
\hline EP & 1,14 & 1,04 & 1,32 & 1,43 & 1,17 \\
\hline $\mathrm{RG}$ & 15,29 & 15,35 & 15,34 & 15,10 & 15,66 \\
\hline $\mathrm{CE}$ & $-0,76$ & 0,09 & $-0,11$ & $-0,13$ & 0,44 \\
\hline
\end{tabular}

1/Pesos econômicos utilizados nos índices de seleção: DPg: desvio-padrão genotípico; CVg: coeficiente de variação genotípico; Iv: índice de variação (relação CVg/Cve); $\mathrm{h}^{2}$ : herdabilidade; e PT: Pesos atribuídos por tentativas $(1 ; 10 ; 20 ; 20 ; 100 ; 100 ; 100 ; 1 ; 20 ; 100 ; 500 ; 1 ; 1000) .{ }^{2 /}$ AP: altura de planta em cm; AE: altura de inserção da primeira espiga em cm; ED: número de espigas doentes; EP: número de espigas atacadas por pragas; RG: rendimento de grãos em kg ha-1; e CE: capacidade de expansão em $\mathrm{mL} \mathrm{g}^{-1}$ 
de ganhos para características de interesse econômico no uso do índice de Mulamba e Mock (1978) com a estratégia de seleção entre e dentro de famílias $\mathrm{S}_{4}$.

Embora o índice de Mulamba e Mock (1978), usando pesos econômicos por tentativas, tenha permitido a obtenção de ganhos satisfatórios para as características estudadas, com base na premissa de Cruz (1990), no melhoramento de plantas, existe dificuldade em se estabelecer pesos econômicos. Conforme propôs Cruz (1990), os pesos econômicos poderiam ser estimados a partir de estatísticas dos próprios dados experimentais. Assim, o coeficiente de variação genotípico $(\mathrm{CVg})$ se constituiria em um bom referencial, pelo fato de ser um parâmetro adimensional e diretamente proporcional à variância genética.

Pelo índice de Mulamba e Mock (1978), embora o uso do CVg tenha favorecido a obtenção de ganhos positivos para $\mathrm{CE}$ e $\mathrm{RG}$, respectivamente, $5,44 \%$ e $2,32 \%$, estes foram menores quando comparados aos obtidos pelo uso dos pesos econômicos atribuídos por tentativas. Contudo, ganhos percentuais favoráveis com boas magnitudes foram identificados para AP, AE, ED e EP (TAB. 4). Deste modo, o emprego do CVg como peso econômico deve ser considerado com atenção na consecução dos próximos ciclos com a população UENF de milho pipoca.

O emprego de um desvio padrão genotípico como peso econômico proporcionou um dos maiores ganhos percentuais para rendimento de grãos $(16,11 \%)$ entre os índices utilizados. Contudo, no que se refere à capacidade de expansão, o ganho percentual foi de apenas $0,30 \%$. Houve, ainda, ganhos não desejáveis para EP, AP e AE (TAB. 4). Assim, pode-se concluir que para o índice de Mulamba e Mock (1978) o uso de um desvio padrão genotípico como peso econômico não constituiu boa alternativa para a seleção de genótipos superiores.

O uso do índice de variação (Iv) e da herdabilidade $\left(h^{2}\right)$ como pesos econômicos foram alternativas interessantes para o uso do índice de Mulamba e Mock (1978). Embora os ganhos percentuais previstos para CE e $\mathrm{RG}$ tenham sido menores do que os previstos usando pesos econômicos atribuídos por tentativas, ganhos desejáveis para AP, AE, ED e EP foram obtidos (TAB. 4).

Por sua vez, os resultados obtidos com o índice de Smith (1936) e Hazel (1943) (TAB. 4) revelaram que apenas para rendimento de grãos os ganhos genéticos preditos foram elevados para todos os pesos econômicos, tendo como valor médio $15,35 \%$. Contudo, ganhos simultâneos desejáveis entre RG e CE não foram observados para quaisquer pesos econômicos atribuídos aleatoriamente. Embora tenham ocorrido ganhos positivos para $\mathrm{CE}$, estes foram de baixa magnitude, a saber: $0,09 \%$ para $\mathrm{CVg}$ e $0,44 \%$ para peso econômico atribuído por tentativas.

As diferenças entre as magnitudes de ganhos genéticos preditos para RG e CE pelo índice de Smith (1936) e Hazel (1943), por diferentes pesos econômicos, também foram constatadas por Santos et al. (2007) e Freitas Júnior et al. (2009) em ciclos anteriores desta população em estudo. Todavia, esses resultados não implicam afirmar que o índice de Smith (1936) e Hazel (1943) não seja adequado para a seleção de progênies na referida população, mas apenas que bons resultados não têm sido obtidos nos últimos ciclos.

O índice de Smith (1936) e Hazel (1943) foi utilizado com sucesso na população de segundo ciclo de seleção recorrente com o objetivo de selecionar progênies superiores em famílias endogâmicas para capacidade de expansão e rendimento de grãos. Os ganhos estimados foram de $17,80 \%$ e $26,95 \%$, respectivamente (DAROS et al., 2004b), bem superiores aos obtidos no primeiro ciclo (10,39\% e 4,69\%, respectivamente), no qual houve seleção direta de progênies de irmãos completos superiores para ambas as características (DAROS et al., 2002).

\section{Conclusões}

A utilização de índices de seleção permitiu a predição de ganhos simultâneos nas duas principais características (CE e RG), sendo que a seleção das famílias superiores, realizada com base no índice de Mulamba e Mock (1978), produziu os maiores ganhos preditos, de $6,01 \%$ para capacidade de expansão e $8,53 \%$ para rendimento de grãos.

\section{Agradecimentos}

À UENF pelo fornecimento da bolsa de estudo; à FAPERJ e ao $\mathrm{CNPq}$, pelo suporte financeiro para a implantação dos experimentos de campo e realização das análises laboratoriais.

\section{Referências}

AMARAL JÚNIOR, A. T. et al. Improvement of a popcorn population using selection indexes from a fourth cycle of recurrent selection program carried out in two different environments. Genetics and Molecular Research, v. 09, n. 01, p. 340-347, 2010.

ARNHOLD, E.; VIANA, J. M. S. Eficiência da seleção dentro de famílias $\mathrm{S}_{4}$ de milho pipoca, visando a obtenção de linhagens. Revista Ceres, v. 54, n. 312, p. 107-111, 2007. 
BRUGNERA, A. et al. Resposta de cultivares de milho pipoca a doses de adubação de semeadura. Revista Ceres, v. 50, n. 290, p. 417-429, 2003.

BRUNSON, A. M. Popcorn breeding. Yearbook Agricultural, n. 01, p. 395-404, 1937.

CARGNELUTTI FILHO, A.; STORCK, L. Estatísticas de avaliação da precisão experimental em ensaios de cultivares de milho. Pesquisa Agropecuária Brasileira, v. 42, n. 01, p.17-24, 2007.

COMSTOCK, R. E.; ROBINSON, H. F. The components of genetic variance in populations of biparental progenies and their use in estimating the average degree of dominance. Biometrics, v. 04, n. 04 , p. $254-266,1948$.

CRUZ, C. D. Aplicação de algumas técnicas multivariadas no melhoramento de plantas. 1990. $188 \mathrm{f}$. Tese (Doutorado em Genética e Melhoramento de Plantas) - Escola Superior de Agricultura "Luiz de Queiroz", Piracicaba.

CRUZ, C. D.; CARNEIRO, P. C. S. Modelos Biométricos Aplicados ao Melhoramento Genético. Viçosa: UFV, 2003. 585 p. v. 2.

DAROS, M. et al. Genetic gain for grain yield and popping expansion in a full-sib recurrent selection in popcorn. Crop Breeding and Applied Biotechnology, v. 02, n. 03, p. 339-344, 2002.

DAROS, M. et al. Genetic gain for grain yield and popping expansion in full-sib recurrent selection in popcorn. Crop Breeding and Applied Biotechnology, v. 02, n. 03, p. 339-344, 2004a.

DAROS, M. et al. Recurrent selection in inbred popcorn families. Scientia Agricola, v. 61, n. 06, p. 609-614, 2004b.

FREITAS JÚNIOR, S. P. et al. Genetic gains in popcorn by full-sib recurrent selection. Crop Breeding and Applied Biotechnology, v. 09, n. 01-07, 2009.

GOMES, F. P. Curso de Estatística Experimental. Piracicaba: USP/ESALQ, 2000. 477 p.

HALlAUER, A. R.; MIRANDA FILHO, J. B. Quantitative genetics in maize breeding. Ames: Iowa State University Press, 1988. 468p.

HAZEL, L. N. The genetic basis for constructing selection indexes. Genetics, v. 28, n. 06, p. 476-490, 1943.

LEONELLO,L.A. F.etal. Características agronômicas e qualidade comercial de cultivares de milho pipoca em alta população. Acta Scientiarum Agronomy, v. 31, n. 02, p. 215-220, 2009.

MENDES DE PAULA, T. O. et al. Magnitude of the genetic base of commercial popcorn and in recommendation in Brazil. Crop Breeding and Applied Biotechnology, v. 10, n. 04 , p. 289-297, 2010.

MIRANDA, G. V. et al. Potencial de melhoramento e divergência genética de cultivares de milho-pipoca. Pesquisa Agropecuária Brasileira, v. 38, n. 06, p.681-688, 2003.
MULAMBA, N. N.; MOCK, J. J. Improvement of yield potential of the Eto Blanco maize (Zea mays L.) population by breeding for plant traits. Egyptian Journal of Genetics and Cytology, v. 01, n. 07, p. 40-51, 1978.

PACHECO, C. A. P. Estimativas de parâmetros genéticos nas populações CMS-42 e CMS-43 de milho pipoca. Pesquisa Agropecuária Brasileira, v. 33, n. 12, p. 1995-2001, 1998.

PACHECO, C. A. P. et al. BRS ANGELA: Variedade de milho pipoca. Comunicado técnico: EMBRAPA/CNPMS, 2000. p. 1-6.

PATERNIANI, E. Melhoramento e produção do milho no Brasil. Campinas: Fundação Cargil, 1980. 650 p.

PEREIRA, M. G.; AMARAL JÚNIOR, A. T. Estimation of genetic components in popcorn based on nested design. Crop Breeding and Applied Biotechnology, v. 71, n. 01, p. 3-10, 2001.

RANGEL, R. M. et al. Genetic parameters in parents and hybrids of circulant diallel in popcorn. Genetics and Molecular Research, v. 07, n. 04, p. 1020-1030, 2008.

ROBBINS, W. A.; ASHMAN, R. B. Parent-offspring popping expansion correlation in progeny of dent $\mathrm{x}$ popcorn crosses. Crop Science, v. 24, n. 01, p. 119-121, 1984.

SANTOS, F. S. et al. Predição de ganhos genéticos por índices de seleção na população de milho pipoca UNB-2U sob seleção recorrente. Bragantia, v. 66, n. 03, p. 391-398, 2007.

SAWAZAKI, E. A cultura do milho pipoca no Brasil. O Agronômico, v. 53, p. 11-13, 2001.

SCAPIM, C. A. et al. Análise dialélica e heterose de populações de milho pipoca. Bragantia, v. 06, n. 03, p. 219-230, 2002.

SCAPIM, C. A. et al. Combining ability of white grain popcorn populations. Crop Breeding and Applied Biotechnology, v. 06, n. 01, p. 136-146, 2006.

SILVA, V.Q. R. et al. Inheritance for economically important traits in popcorn from distinct heterotic groups by Hayman's diallel. Cereal Research Communications, v. 38, n. 02, p. 273-285, 2010a.

SILVA, V. Q. R. et al. Combining ability of tropical and temperate inbred lines of popcorn. Genetics and Molecular Research, v. 09, n. 03, p. 1742-1750, 2010 b.

SIMON, G. A. et al. Depressão por endogamia em populações de milho pipoca. Bragantia, v. 63, n. 01, p. 55-62, 2004.

SMITH, H. F. A discriminant function for plant selection. Annals of Eugenics, v. 07, p. 240-250, 1936.

VIEIRA, A. A. et al. Heterotic parametrization for economically important traits in popcorn. Acta Scientiarum. Agronomy, v. 31, n. 03, p. 411-419, 2009.

VILARINHO, A. A. et al. Seleção de progênies endogâmicas $\mathrm{S}_{1} \mathrm{e}$ $\mathrm{S}_{2}$ em um programa de melhoramento intrapopulacional de milho pipoca. Acta Scientiarum, v. 24, n. 05, p. 1419-1425, 2002. 